\title{
BMJ Open Mapping evidence on the implementation of the WHO's collaborative framework for the management of tuberculosis and diabetes: a scoping review protocol
}

\author{
Rita Quist-Therson (D) , Desmond Kuupiel (D) , Khumbulani Hlongwana
}

To cite: Quist-Therson R, Kuupiel D, Hlongwana $\mathrm{K}$. Mapping evidence on the implementation of the WHO's collaborative framework for the management of tuberculosis and diabetes: a scoping review protocol. BMJ Open 2020;10:e033341. doi:10.1136/ bmjopen-2019-033341

- Prepublication history and additional material for this paper are available online. To view these files, please visit the journal online (http://dx.doi. org/10.1136/bmjopen-2019033341).

Received 09 August 2019 Revised 26 December 2019 Accepted 09 January 2020

Check for updates

(C) Author(s) (or their employer(s)) 2020. Re-use permitted under CC BY-NC. No commercial re-use. See rights and permissions. Published by BMJ.

Discipline of Public Health Medicine, School of Nursing and Public Health, College of Health Sciences, University of KwaZuluNatal, Durban, South Africa

Correspondence to Rita Quist-Therson; suhyini@gmail.com

\section{ABSTRACT}

Introduction The emergence of tuberculosis (TB) and diabetes mellitus (DM) coepidemic threatens the gains made in fighting the prevalence of these two diseases. As a result, in 2011, WHO and the International Union Against Lung Disease launched a framework to address the growing TB-DM coepidemic across the world. The aim of the proposed review study is mapping evidence on the implementation of the $\mathrm{WHO}$ collaborative framework for the management of TB-DM using a scoping review. Methodology and analysis This study will map literature on the global implementation of the WHO collaborative framework for the management of TB-DM, using Arksey and 0'Malley's scoping review framework. An extensive literature search for the peer-reviewed articles, grey literature, unpublished studies, thesis, studies in the press and a list of references from the selected studies will be conducted to find eligible studies. PubMed, Google Scholar, Web of Science, Science Direct, the EBSCOhost platform (Academic search complete, health source: nursing/ academic edition, CINAHL with full text) and the WHO library will be used to source literature. The researcher will perform title screening of articles using keywords in the databases, and two independent reviewers will then screen abstracts and full articles. The screening will be guided by the inclusion and exclusion criteria. The Mixed Method Appraisal Tool V.2018 will be used to examine the quality of studies to be included. The findings will be analysed using the thematic content analysis approach and the results presented in the form of a narrative report. Ethics and dissemination The study did not require ethics approval because it is a scoping review protocol. Findings from this study will be disseminated by print and electronic mediums.

\section{BACKGROUND}

The association between tuberculosis (TB) and diabetes is gaining global traction, as more evidence is increasingly being documented on the adverse effect of one disease on the other. ${ }^{1}$ The emergence of TB and diabetes mellitus coepidemic (TB-DM) ${ }^{2}$ makes the diagnostic accuracy and management of the
Strengths and limitations of this study

- The use of Arksey and 0'Malley's framework to guide the literature search process will ensure a clear methodological and transparent process that can be replicated.

- Findings from this research will be of value for policy-makers and health workers in countries implementing the WHO's Collaborative Framework for the management of tuberculosis and diabetes comorbidity.

- To the best of our knowledge, this study will be the first to systematically review evidence on the implementation of the WHO's Collaborative Framework for the Management of Tuberculosis and Diabetes.

- An important limitation of this study will be language since this review will only include literature published in English.

two diseases, challenging to achieve, thereby necessitating a rethink on the vertical nature of TB and DM management. ${ }^{12}$

The coepidemic of TB and DM has serious public health consequences, mainly because TB infects one-third of the global population. ${ }^{3}$ $\mathrm{DM}$ is one of the top four non-communicable diseases, which in 2015, caused an estimated 3 million deaths, worldwide. ${ }^{3}$ Research demonstrates that approximately $80 \%$ and $90 \%$ of DM and TB cases, respectively, occur in low-income and middle-income countries (LMICs). ${ }^{4}$ Chronic conditions, such as DM, weaken the immune system and leaves populations susceptible to and at increased risk for TB infection. ${ }^{5}$ Having DM triples the risk of developing TB, and the risk of TB is higher in people with DM than in the general population. ${ }^{6}$ The clinical manifestations of TB-DM coepidemic adversely affect the treatment, increase the risk of death and the likelihood of $\mathrm{TB}$ recurrence. ${ }^{7}$ WHO estimated that 
about $15 \%$ of people with TB, globally, can be linked to DM as well. ${ }^{4}$ It is estimated that in 22 countries which carry $80 \%$ of the global TB burden, TB arising from DM increased from $10 \%$ in 2010 to $15 \%$ in $2013 .{ }^{8}$ Nine of the top 10 countries with high TB-DM incidence are from the LMICs. ${ }^{8}$

In 2011, an approach to curb the rising TB-DM coepidemic, otherwise known as a Collaborative Framework for the Management of TB and DM, was launched by WHO and International Union Against Lung Disease. ${ }^{9}$ This collaborative framework was developed to guide the care, prevention and control of TB and DM in signatory countries across the world. ${ }^{9}$ The aim was to outline TB-DM comanagement strategies for use by policy-makers and implementers in order to reverse the TB-DM coepidemic. $^{2}$ The framework is designed to complement the core systems setup for the prevention and management of both diseases and focuses on a three-broad objectives, namely: to establish mechanisms of collaboration between $\mathrm{TB}$ and $\mathrm{DM}$ programmes, to improve detection and management of TB in patients with DM and to improve detection and management of DM in patients with TB. ${ }^{9}$ To achieve these objectives, the framework proposed 12 interventions, which include: 'exploration of screening for active TB on broader indications (eg, in all people diagnosed with diabetes, regardless of symptoms), as part of the research agenda for improved TB diagnosis among people with diabetes,' 'provision of the management of diabetes in patients with TB in line with existing guidelines,' and the 'establishment of joint coordination at regional, district and/or local levels (sensitive to country-specific factors), with representation from all relevant stakeholders.'

Further implementation research is encouraged to gather evidence for countries to adopt these recommendations to their healthcare systems. ${ }^{9}$ This scoping review, therefore, aims at mapping evidence on the implementation of the WHO's collaborative framework for the management of TB and DM. Results from this scoping review are expected to demonstrate current data on the state of implementation of the WHO's TB-DM collaborative framework globally, thereby revealing gaps in current literature, and ultimately inform the refinement of questions for further primary research.

\section{METHOD}

\section{Study design}

This study will use a scoping review methodology, guided by Arksey and O'Malley's framework ${ }^{10}$ to map literature on the implementation of the WHO collaborative framework for the management of TB-DM. Arksey and O'Malley's framework ${ }^{10}$ gives five clear steps to be used to explore core concepts in a specific research area. These steps are identifying the research question, identifying relevant studies, study selection, charting the data, collating, summarising and reporting the results. This process is expected to help identify the existing evidence
Table 1 PCC framework for defining the eligibility of the studies for the primary research question

\begin{tabular}{ll}
\hline P-Population & $\begin{array}{l}\text { Individuals with TB } \\
\text { Individuals with DM }\end{array}$ \\
C-Concept & $\begin{array}{l}\text { WHO Collaborative Framework } \\
\text { Implementation }\end{array}$ \\
C-Context & Globally \\
\hline
\end{tabular}

DM, diabetes mellitus; TB, tuberculosis.

in the research area. In this study, the Preferred Reporting Items for Systematic Reviews and Meta-Analyses extension for Scoping Reviews (PRISMA-ScR) will be used to report the results from the screening process (online supplementary file). ${ }^{11}$

\section{Identify research questions}

Main research question: What is the evidence on the implementation of the WHO's TB-DM collaborative framework?

Subquestions:

1. What is the evidence of DM detection and management in patients with TB?

2. What is the evidence of TB detection and management in patients with DM?

3. What is the evidence of collaboration between TB and DM programmes?

4. What is the evidence of a referral system for patients with DM suspected to have TB for diagnosis and management?

5. What is the evidence of a referral system for patients with $\mathrm{TB}$ suspected to have DM for diagnosis and management?

\section{Eligibility of the research question}

This scoping review will use the Population, Concept, Context Mnemonic (table 1) to determine the eligibility of the primary research question. ${ }^{10}$

\section{Identifying relevant studies}

We intend to conduct a scoping review to map literature on the implementation of the TB-DM collaborative framework, globally. This will be done by searching electronic databases for peer-reviewed articles, grey literature and reference lists of included articles. The databases will include the EBSCOhost platform (Academic search complete, health source: nursing/academic edition, CINAHL with full text), PubMed, Google Scholar, Scopus and WHO library databases. Grey literature will be accessed by searching for policy documents, treatment guidelines and reports by ministries of health, health agencies either through their websites or links.

We will search for articles using keywords and Boolean terms AND/OR. Additionally, a combination of the appropriate Medical Subject Headings terms will be included in the search. In conducting the "electronic search, the proposed combinations of keywords to be used include: 


\begin{tabular}{|c|c|c|c|}
\hline Date & Database & Keywords & $\begin{array}{l}\text { Search } \\
\text { results }\end{array}$ \\
\hline 27/03/2019 & PubMed & $\begin{array}{l}\text { ((("tuberculosis"[MeSH Terms] OR "tuberculosis"[All Fields]) OR ("diabetes } \\
\text { mellitus"[MeSH Terms] OR ("diabetes"[All Fields] AND "mellitus"[All Fields]) } \\
\text { OR "diabetes mellitus"[All Fields] OR "diabetes"[All Fields] OR "diabetes } \\
\text { insipidus"[MeSH Terms] OR ("diabetes"[All Fields] AND "insipidus"[All } \\
\text { Fields]) OR "diabetes insipidus"[All Fields])) AND implementation[All Fields]) } \\
\text { AND (collaborative[All Fields] AND framework[All Fields]) }\end{array}$ & 36 \\
\hline
\end{tabular}

'Diabetes,' 'Type 2 Diabetes,' 'Tuberculosis,' 'Comorbidity,' 'Implementation,' 'Framework.' Studies will be identified by searching literature that was published in English from August 2011 to December 2019. With type 2 diabetes contributing $90 \%-95 \%$ of all diabetes cases globally and sharing socioeconomic, environmental, and behavioural factors with TB, this study will exclude articles on type 1 diabetes. ${ }^{12}$ Searches will be documented, detailing the date, search engine, keywords and the number of publications retrieved. The search strategies will be piloted to check the suitability of the selected databases and keywords. An illustration of how the electronic data search will be recorded is shown in table 2 .

\section{Study selection and eligibility criteria}

The eligibility criteria will be developed to ensure that specific information relating to the research questions is included in the studies.

\section{Inclusion criteria}

We will include studies that meet the following criteria:

- Evidence of the study among TB and DM populations.

- Evidence of study conducted from 2011 to the last search date.

- Present evidence of bidirectional screening for TB and DM.

- Present evidence of mechanisms of collaboration in the management of TB and DM.

\section{Exclusion criteria}

This will include the following:

- Studies on type 1 diabetes.

- Studies before 2011.

- Studies on TB/HIV.

The eligibility criteria will guide the researcher in conducting the title screening. Studies that qualify will be compiled into a library using the Endnote reference management software. Duplicated articles will be deleted from the EndNote library. The final list of compiled articles will be shared with two other reviewers who will conduct both abstract and full screening into two categories 'excluded' and 'included' according to the inclusion criteria detailed above. The service of the University of KwaZulu-Natal Libraries will be sought to source articles that are not available online, or the full text may be requested from the authors. During the abstract screening stage, categorisation by the two reviewers will be compared. In the event of any disagreement, the reviewers will discuss until an agreement is reached. At the full-text stage, a third reviewer will be engaged as a decider on any unresolved disputes. Details of the process: date of search, database, keywords, number of studies and number of eligible studies will be comprehensively documented.

Screening of the results and reporting will follow the PRISMA-ScR. ${ }^{11}$

\section{Charting the data}

To extract information in line with the aim of this study, an analytical method will be used. We will develop a form electronically, using google forms, pretest it and use feedback to refine the tool. During data extraction, all articles reviewed and excluded will be tracked (table 3 ).

\section{Collating, summarising and reporting of results}

When the extraction of data is completed, the results from existing studies will be summarised and presented in a narrative account. This summary will be analysed using thematic content analysis. Data extracted will be structured around the following outcomes: Bidirectional screening of TB and DM, mechanisms for TB and DM collaborative activities and comanagement of TB/ DM comorbidity. Emerging themes will also be coded using NVivo software V.10. ${ }^{13}$ The themes emerging from the analysis will be examined to determine whether they address the research questions. Furthermore, the researcher will explore the linkages between the findings, study aim and the implications for future research, policy and practice.

\section{Quality appraisal}

The quality of the studies will be appraised using the Mixed Method Appraisal Tool V.2018. ${ }^{14}$ The tool is used to assess the risk of bias of the included studies. The tool will be used to assess the selected articles under the following groupings: the appropriateness of the aim of the study, adequacy of methodology, study design, data collection, data analysis, the presentation of findings, authors' discussions and conclusions. Scores will be allocated to determine the quality of the included studies. The quality of the selected articles will be determined from the examination of the aspects mentioned above.

\section{Patient and public involvement}

The conception, design and planning of this study did not directly include patients or the public. 
Table 3 Data charting form

\section{Author and date}

Study title
Journal full reference
Aims or research question
Participant characteristics
Recruitment context (eg, where people were
recruited)
Sampling method
Study design
Theoretical background
Data collection (what data collection
methods were used?)
Data analysis (how was the data analysed?)
Most relevant findings
Conclusions
Comments

\section{Ethics and dissemination}

The study did not require ethics approval because it is a scoping review protocol. Findings from this study will be disseminated by print and electronic mediums.

\section{DISCUSSION}

This scoping review is aimed at mapping existing evidence and summarise the findings as presented across the studies on the implementation of the WHO's TB/DM collaborative framework, globally. TB is still among the top causes of mortality globally. The number of people living with $\mathrm{DM}$ and $\mathrm{TB}$ is expected to rise to as high as 592 million by 2035, globally. Therefore, TB-DM comorbidity represents a critical public health concern. ${ }^{12} 15$ The continued rise of TB-DM comorbidity necessitated the development of the framework to manage this epidemic. However, evidence on the extent of implementation of this framework in various settings is limited. ${ }^{9}$ The framework presents several recommendations, including bidirectional screening, as a critical strategy in the diagnosis and management of TB-DM comorbidity. ${ }^{9}$

There is no consensus on what is likely to yield better health outcomes. Some studies propose that screening all patients with TB for DM in high-income countries and screening all patients with DM for TB in low-income settings would yield better results. ${ }^{16}$ Conversely, Di Gennaro et $a l^{16}$ disagree but consider the screening for $\mathrm{DM}$ in patients with TB in low-income settings as sufficient. Further evidence is needed to support the feasibility of the recommendations, so that appropriate guidelines for the different settings can be developed. ${ }^{9}$ This study will focus on literature available from August 2011 to December 2019, in order to add to the body of evidence regarding critical issues on the state of the framework implementation, worldwide. Findings from this study are expected to be useful to the implementation process by various countries.

A preliminary search suggests that accumulated literature since the launch of the framework in 2011 is limited, ${ }^{9}$ especially given that publications on type 1 diabetes will be excluded since this condition does not share socioeconomic, environmental and behavioural factors with TB. ${ }^{17}$ The findings of the scoping review will generate valuable information necessary to support initiatives aimed at curtailing the rising tide of TB and DM comorbidity. ${ }^{2}$ The results of this study may interest policy-makers and stakeholders involved in the implementation of the prevention, care and control strategies for TB and DM, including the health systems strengthening. Also, the findings of this study will be of interest to researchers, as it will highlight the gaps in evidence that may require further empirical investigation.

Acknowledgements We are grateful to the University of KwaZulu-Natal, College of Health Sciences, for providing us with vital research resources to complete this study protocol. We would also like to appreciate the staff of the Department of Public Health Medicine, the University of KwaZulu-Natal for their diverse support.

Contributors RQ-T conceptualised the study and wrote the protocol. DK and KH contributed to the writing and critically reviewed the draft protocol. RQ-T wrote the final manuscript and all authors approved the final draft.

Funding This study is funded by the University of KwaZulu-Natal, College of Health Sciences Research Scholarship.

Competing interests None declared.

Patient consent for publication Not required.

Provenance and peer review Not commissioned; externally peer reviewed.

Open access This is an open access article distributed in accordance with the Creative Commons Attribution Non Commercial (CC BY-NC 4.0) license, which permits others to distribute, remix, adapt, build upon this work non-commercially, and license their derivative works on different terms, provided the original work is properly cited, appropriate credit is given, any changes made indicated, and the use is non-commercial. See: http://creativecommons.org/licenses/by-nc/4.0/.

\section{ORCID iDs}

Rita Quist-Therson http://orcid.org/0000-0001-6508-6915

Desmond Kuupiel http://orcid.org/0000-0001-7780-1955

\section{REFERENCES}

1 Pizzol D, Di Gennaro F, Chhaganlal KD, et al. Tuberculosis and diabetes: current state and future perspectives. Trop Med Int Health 2016;21:694-702.

2 Kapur A, Harries AD, Lönnroth K, et al. Diabetes and tuberculosis co-epidemic: the Bali Declaration. Lancet Diabetes Endocrinol 2016;4:8-10.

3 Kapur A, Harries AD. The double burden of diabetes and tuberculosis - public health implications. Diabetes Res Clin Pract 2013;101:10-19.

4 Mbanya JCN, Motala AA, Sobngwi E, et al. Diabetes in sub-Saharan Africa. Lancet 2010;375:2254-66.

5 Workneh MH, Bjune GA, Yimer SA. Prevalence and associated factors of tuberculosis and diabetes mellitus comorbidity: a systematic review. PLoS One 2017;12:e0175925.

6 Harries AD, Kumar AMV, Satyanarayana S, et al. Diabetes mellitus and tuberculosis: programmatic management issues. Int J Tuberc Lung Dis 2015;19:879-86.

7 Reid MJA, McFadden N, Tsima BM. Clinical challenges in the comanagement of diabetes mellitus and tuberculosis in southern Africa. Journal of Endocrinology, Metabolism and Diabetes of South Africa 2013;18:135-40.

8 Lönnroth K, Roglic G, Harries AD. Improving tuberculosis prevention and care through addressing the global diabetes epidemic: from evidence to policy and practice. Lancet Diabetes Endocrinol 2014;2:730-9. 
9 WHO. Collaborative framework for care and control of tuberculosis and diabetes. Geneva: World Health Organization, 2011.

10 Arksey H, O'Malley L. Scoping studies: towards a methodological framework. Int J Soc Res Methodol 2005;8:19-32.

11 Tricco AC, Lillie E, Zarin W, et al. PRISMA extension for scoping reviews (PRISMA-ScR): checklist and explanation. Ann Intern Med 2018;169:467-73.

12 Harries AD, Kumar AMV, Satyanarayana S, et al. Addressing diabetes mellitus as part of the strategy for ending TB. Trans $R$ Soc Trop Med Hyg 2016;110:173-9.

13 Castleberry A. NVivo 10 [software program]. Version 10. QSR International; 2012. Am J Pharm Educ 2014;78:25.
14 Hong QN, Pluye P, Bartlett G, et al. Mixed methods appraisal tool (MMAT), version 2018, 2018.

15 The Global Fund to Fight AIDS TaM. Best practices on TB case finding and treatment reflections and lessons from West and central Africa and beyond, 2018.

16 Di Gennaro F, Marotta C, Antunes M, et al. Diabetes in active tuberculosis in low-income countries: to test or to take care? Lancet Glob Health 2019;7:e707.

17 Anand T, Kishore J, Isaakidis P, et al. Integrating screening for non-communicable diseases and their risk factors in routine tuberculosis care in Delhi, India: a mixed-methods study. PLoS One 2018;13:e0202256. 